\title{
Resistência à abrasão, dureza Janka e a correlação com outras propriedades em 14 espécies de madeiras tropicais brasileiras com potencial para utilização em pisos de madeira
}

\author{
Divino Eterno Teixeira ${ }^{1 \star}$, Laís Ernesto Cunha², Peter Wimmer ${ }^{1}$, Ariel de Andrade ${ }^{3}$ \\ ${ }^{1}$ Serviço Florestal Brasileiro - SFB, Brasília, DF, Brasil. \\ ${ }^{2}$ Universidade de Brasília, Brasília, DF, Brasil. \\ ${ }^{3}$ Associação Nacional dos Produtores de Pisos de Madeira - ANPM, Brasília, DF, Brasil.
}

\begin{abstract}
RESUMO Quatorze espécies de madeiras tropicais brasileiras foram coletadas em 2011 na UPA-06, área de exploração sob plano de manejo florestal sustentável (PMFS), no município de Almeirim/Monte Dourado - Pará, Brasil. Essas madeiras foram estudadas objetivando caracterizar as propriedades físicas e mecânicas e determinar o potencial para uso como pisos de madeira maciça. Para o teste de resistência à abrasão, as amostras foram cortadas em corpos de prova e submetidas ao ensaio de taxa de desgaste no Abrasímetro Taber com roda H18, 500 ciclos e carga de $1000 \mathrm{~g}$. No total, foram testados 84 corpos de prova, seis de cada espécie. A variação de perda de massa observada, antes e após o teste, foi usada no cálculo da taxa de desgaste à abrasão. As taxas variaram de 15,45 a 40,13 mg/100 ciclos para as espécies Sapucaia (Lecythis pisonis Cambess) e Timborana (Piptadenia gonoacantha (Mart.)), respectivamente. As correlações entre resistência à abrasão e densidades (básica e aparente), dureza Janka e extração de prego variaram de $2,2 \%$ a $58,9 \%$. As maiores correlações da resistência à abrasão foram encontradas com: densidade aparente $(-0,424)$, dureza Janka de topo $(-0,589)$ e extração de pregos de face $(-0,578)$, todos na condição seca (a $12 \%$ de teor de umidade).
\end{abstract}

Palavras-chave: Densidade aparente; densidade básica; abrasímetro Taber; variação de massa; taxa de desgaste.

\section{Resistance to abrasion, Janka hardness and the correlation with other properties in 14 species of Brazilian tropical wood with potential for use in wood floorings}

\begin{abstract}
Fourteen Brazilian tropical wood species were collected in UPA-06, area under sustainable forest management plans (SFMP), in the municipality of Almeirim/Monte Dourado - Pará, Brazil. These wood species were studied with the purpose of characterizing the physical and mechanical properties and determine their potential for use as solid wood flooring. For the abrasion resistance test, the wood samples were cut into specimens and subjected to the wear test on the Taber Abrasimeter with $\mathrm{H} 18$ wheel, 500 cycles and load of $1000 \mathrm{~g}$. A total of 84 specimens were tested, six of each species. The variation of the mass loss observed, before and after the test, was used in the calculation of the wear rate. The results ranged from 15.45 $\mathrm{mg} / 100$ cycles to $40.13 \mathrm{mg} / 100$ cycles for the species Sapucaia (Lecythis pisonis Cambess) and Timborana (Piptadenia gonoacantha (Mart.)), respectively. The correlations between abrasion resistance and density, Janka hardness and nail withdrawal ranged from $2.2 \%$ to $58.9 \%$. The highest correlations of abrasion resistance were found with apparent density ($0.424)$, end Janka hardness (-0.589) and side nail extraction (-0.578), all at $12 \%$ moisture content.
\end{abstract}

Keywords: Density; Taber abrasimeter; mass variation; wear rate. 


\section{Introdução}

O piso de madeira sólida acabado (assoalho, parquet, tacos, outros) é um produto de maior valor agregado (PMVA), que necessita da adoção de estratégias e ações para o seu adequado desenvolvimento e divulgação. Há, portanto, uma necessidade de inverter essa tendência para que ao longo dos anos a quantidade exportada de madeira serrada brasileira sem beneficiamento diminua e aumente a de pisos maciços pré-acabados e acabados para o mercado externo (ANDRADE, 2015).

Um requisito básico para se determinar se uma espécie de madeira é adequada para um uso determinado é o conhecimento e a análise de suas propriedades físicomecânicas por meio de testes regulados por Normas, que permitam a comparação com outras espécies ou produtos similares. Os principais ensaios de avaliação para o desgaste em pisos estão condicionados à resistência à abrasão superficial, dureza Janka e densidade da madeira, onde o mesmo está sujeito a movimentos de objetos ou de pessoas. Esses fatores de desgaste, servem para se determinar a vida útil e potencial de aplicação de um material para piso (CADEMARTORI et al., 2016; PAES et al. 2010; SILVA; BITTENCOURT, 2002).

O ensaio de abrasão é um teste de baixa complexidade, que pode ser conduzido sem maiores problemas, porém, deverá ser realizado por um operador experiente, com limpeza dos rebolos durante o teste buscando evitar o empastamento, o qual pode influenciar diretamente nos resultados.

Com objetivo de conhecer o comportamento de materiais vegetais alternativos à madeira de lei, Silva; Bittencourt (2002) testaram a resistência à abrasão do bambu gigante (Dendrocalamus giganteus), da madeira laminada e colada de Jatobá (Hymenaea sp.) e do Eucalipto (Corymbia citriodora), seguindo a Norma American Society for Testing and
Materials - ASTM D4060-14 (2014) com a utilização do Abrasímetro Taber. Comprovou-se que, em relação a perda de massa, ao submeter os corpos de prova a 500 ciclos de abrasão, o bambu gigante e o eucalipto apresentaram maior resistência em relação ao laminado de Jatobá.

Ncube (2008) discutiu o uso de um dispositivo abrasivo como alternativa à difícil obtenção de equipamentos para testes físicos em madeiras a fim de estudar a resistência à abrasão de Blackwood (Acacia melanoxylon), Mahogany (Khaya sp.), Poplar (Populus sp.) e Iroko (Chlorophora excelsa), e compará-la com a dureza Janka dessas espécies. Neste estudo de Ncube (2008), as amostras foram submetidas a 100 ciclos de abrasão, com velocidade de rotação de nove ciclos por minuto. Os resultados demonstraram que nem o valor de dureza Janka, nem a densidade básica da madeira podem explicar o aumento da resistência ao desgaste abrasivo e, de acordo com o autor, isto seria controlado por certos compostos encontrados nas cavidades da madeira. No entanto, a classificação da madeira de lei por classes de tráfego de pisos, foi semelhante para valores de dureza e resistência à abrasão, indicando o sucesso do teste de resistência, obtendo novas possibilidades para o uso de espécies alternativas.

A fim de agregar valor comercial a onze espécies madeireiras da Amazônia e, determinar sua viabilidade para utilização em pisos, Lopes (2012) realizou o ensaio de desgaste à abrasão de acordo com a Norma ASTM D4060-14 (2014), com uso de um abrasímetro Taber com rebolo H18, velocidade de 60 rpm, carga de 500 g e 500 ciclos. Foram testados 55 corpos de prova e, periodicamente, verificou-se que algumas madeiras liberavam grande quantidade de resíduos nos rebolos. Não foram encontradas correlações satisfatórias entre os resultados obtidos nos ensaios de resistência à abrasão e os resultados de flexão estática, compressão paralela e dureza Janka e, portanto, concluiu-se que não foi possível desenvolver uma equação que estime a 
resistência à abrasão em função das demais propriedades mecânicas.

O uso de madeiras tropicais para pisos sólidos no Brasil está concentrado em espécies, tais como Sucupira (Bowdichia sp.), Grápia (Apuleia leiocarpa), Cumarú (Dipteryx odorata), Ipê (Handroanthus sp.), Tauari (Couratari spp.), Maçaranduba (Manilkara huberi), Pau-marfim (Balfourodendron riedelianum), Jatobá (Hymenaea spp.), entre outras. A disponibilização de informação sobre uma maior diversidade de espécies para pisos diminui a pressão da exploração florestal em poucas espécies e aumenta a oferta de madeiras para esse fim, além de proporcionar mais eficiência e aproveitamento do manejo florestal (ANDRADE, 2015; CADEMARTORI et al., 2016).

Esse trabalho teve como objetivo, caracterizar as propriedades físicas e mecânicas da madeira de 14 espécies tropicais brasileiras a fim de determinar o potencial para uso como pisos de madeira maciça.

\section{Material e Métodos}

As 14 espécies de madeiras selecionadas para o projeto, conforme frequência na floresta e interesse comercial, foram coletadas no município de Almeirim/Monte Dourado-Pará, Brasil, em setembro de 2011 na UPA-06 de área de plano de manejo florestal sustentável (PMFS). Foram abatidas três árvores de cada espécie e retiradas duas vigas de $17 \times 17 \times 250 \mathrm{~cm}$ de cada, as quais foram enviadas para o Laboratório de Produtos Florestais (LPF), do Serviço Florestal Brasileiro (SFB), em Brasília, onde o trabalho foi realizado. A Tabela 1 apresenta as 14 espécies estudadas neste trabalho.

As vigas de madeira foram processadas na carpintaria do LPF, onde foi realizado o desdobro em serra circular e em desengrossadeira, seguidas de aplainamento e lixa, com dimensionamento das medidas de espessura e largura dos corpos de prova para testes. Em seguida, os corpos de prova foram armazenados na sala de climatização, em condições controladas de temperatura $20 \pm 3{ }^{\circ} \mathrm{C}$ e umidade relativa $65 \pm$

Tabela 1. Lista das espécies de madeiras.

Table 1. List of wood species.

\begin{tabular}{c|l|l|c}
\hline Código & \multicolumn{1}{|c|}{ Nome Popular } & \multicolumn{1}{c|}{ Nome científico } & $\begin{array}{c}\text { Densidade básica } \\
\left(\mathrm{g} / \mathrm{cm}^{3}\right)\end{array}$ \\
\hline A & Angelim vermelho & Dinizia excelsa Ducke & 0,88 \\
\hline B & Itaúba amarela & Mezilaurus itauba (Meisn) exMez & 0,76 \\
\hline C & Cedrinho & Erisma uncinatum Warm. & 0,53 \\
\hline D & Cupiúba & Goupia glabra Aubl. & 0,71 \\
\hline E & Pequiá & Caryocar villosum (Aubl.) Pers. & 0,73 \\
\hline F & Angelim da mata & Hymenolobium excelsum Ducke & 0,63 \\
\hline G & Maparajuba & Manilkara bidentata (A. DC.) A. Chev & 0,80 \\
\hline H & Timborana & Piptadenia gonoacantha (Mart.) & 0,74 \\
\hline I & Sucupira preta & Bowdichia nítida Spruce ex Benth. & 0,80 \\
\hline J & Tachi preto & Tachigali myrmecophila Ducke & 0,60 \\
\hline K & Tanibuca & Terminalia amazonica (J.F. Gmel) & 0,78 \\
\hline L & Mandioqueira-escamosa & Qualea paraensis Ducke & 0,65 \\
\hline M & Sapucaia & Lecythis pisonis Cambess & 0,86 \\
\hline N & Jarana amarela & Lecythis poiteaui O. Berg & 0,86 \\
\hline
\end{tabular}


$5 \%$, até atingirem teor de umidade de equilíbrio (TUE) de $12 \%$.

Para o teste de resistência à abrasão os corpos de prova foram dimensionados em 100x100x4 mm. Foram testados 84 corpos de prova, seis de cada espécie, de forma que as madeiras de maior densidade foram as últimas a serem avaliadas, diminuindo assim, o desgaste dos rebolos.

As amostras foram pesadas em uma balança analítica (0,0001 g de precisão) e, em seguida, fixadas nos discos rotativos do abrasímetro Taber (Figura 1), com roda H18, e submetidas à 500 ciclos e carga de 1000 g. Durante esse processo, acontece um "empastamento" dos rebolos, que foram limpos a cada 250 ciclos, com utilização somente de lixa e pincel.

A variação de massa, observada entre as pesagens inicial e após o teste, foi determinada para o cálculo da taxa de desgaste (TD), de acordo com a Norma Associação Brasileira De Normas Técnicas -ABNT NBR 14535-2008 (2008) e a Equação 1:

$$
\mathrm{TD}=100 \times \frac{\mathrm{A}-\mathrm{B}}{\mathrm{C}}
$$

Onde: TD: taxa de desgaste, em mg/100 ciclos; A: massa da amostra antes do ensaio, em mg; B: massa da amostra após o ensaio, em mg; C: número de ciclos de abrasão realizados.

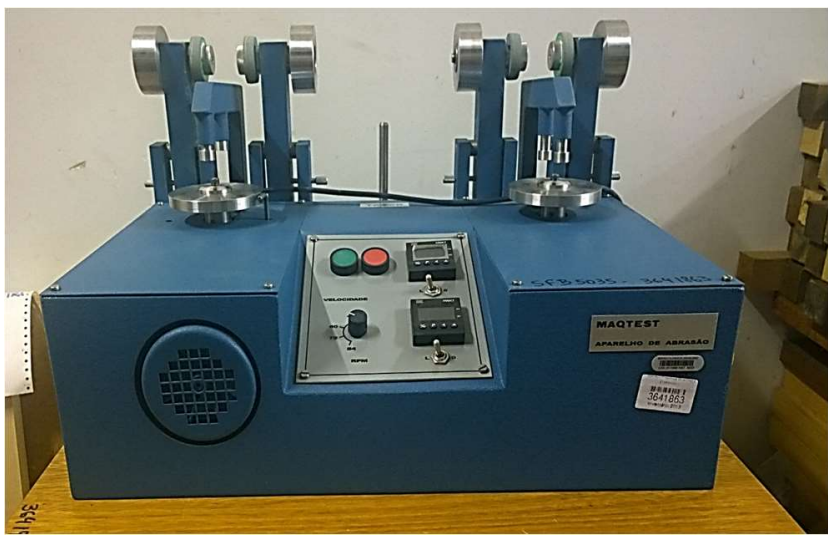

Figura 1. Abrasímetro Taber utilizado no teste de abrasão. Figure 1. Taber Abrasimeter used in the abrasion test.
Os ensaios de dureza Janka, extração de prego e densidades, básica e aparente (a 12\% de umidade), de cada corpo de prova, foram conduzidos de acordo com a Norma ASTM D143-14 (2014).

A determinação da dureza Janka foi realizada em corpos de prova de 50x50x150 mm, com a penetração de uma semiesfera de $11.3 \mathrm{~mm}$ de diâmetro, em máquina universal de ensaios USM-600 com capacidade de $600 \mathrm{kN}$ e velocidade de carregamento de $6 \mathrm{~mm} / \mathrm{min}$. O cálculo da dureza Janka foi obtido pela carga requerida para penetrar a semiesfera de área igual a $1 \mathrm{~cm}^{2}$.

Para o ensaio de extração de prego, na mesma máquina universal, foi utilizado prego com diâmetro de $2,5 \mathrm{~mm}$, o qual deve penetrar $32 \mathrm{~mm}$ na madeira. Este teste, a exemplo do de dureza Janka, foi realizado nas faces e nos topos das amostras. A extração de prego foi determinada pela carga máxima para retirada do mesmo da madeira.

No teste de densidade (básica e aparente), conforme a Norma ASTM D143-14 (2014), foram usadas amostras de 20x20x100 mm e determinado seu valor usando a Equação 2:

$$
\text { Densidade }=\frac{\mathrm{m}}{\mathrm{V}}
$$

Onde: m:massa da amostra, em g; V:volume da amostra, em $\mathrm{cm}^{3}$

Os dados adquiridos nos ensaios de densidade básica, resistência à abrasão e dureza Janka foram submetidos à análise estatística, conduzida com o auxílio do software SPSS plus versão 22.0 e, os resultados obtidos, foram analisados com base no teste de médias de Tukey HSD a 5\% de significância.

\section{Resultados e Discussão}

\section{Resistência à abrasão}

Os resultados médios dos ensaios por espécie de madeira estão apresentados na Figura 2. 
As espécies Sapucaia (M), Angelim vermelho (A) e Sucupira preta (I), com 15,45, 15,48 e 18,13 mg/100ciclos, respectivamente, foram as espécies de menor desgaste abrasivo, e a Timborana a de maior desgaste. Para o Angelim vermelho (A) somente a taxa não é um bom indicador, uma vez que a espécie libera um odor desagradável com a presença de alta umidade e, portanto, deve-se tomar o cuidado de ser recomendado seu uso após secagem apropriada.

Analisando os resultados desejados para características de pisos de madeira, ou seja, alta densidade básica, alta dureza Janka de face na condição seca (12\% de teor de umidade) e baixa taxa de desgaste na abrasão, destingem-se as espécies Angelim vermelho (A), Sucupira preta (I), Sapucaia (M) e Jarana amarela $(\mathrm{N})$. Do outro lado sobressaem-se duas espécies, Tanibuca (K) e Maparajuba (G), por apresentarem alta densidade e dureza Janka, porém numa faixa de menor resistência à abrasão. Apesar de apresentar densidade básica e dureza Janka de face centrados na mediana, a espécie Timborana $(\mathrm{H})$ destaca-se pela menor resistência à abrasão.

Valores de desgaste à abrasão em onze espécies madeireiras da Amazônia, entre 8,8 e 35,9 mg/100ciclos, são
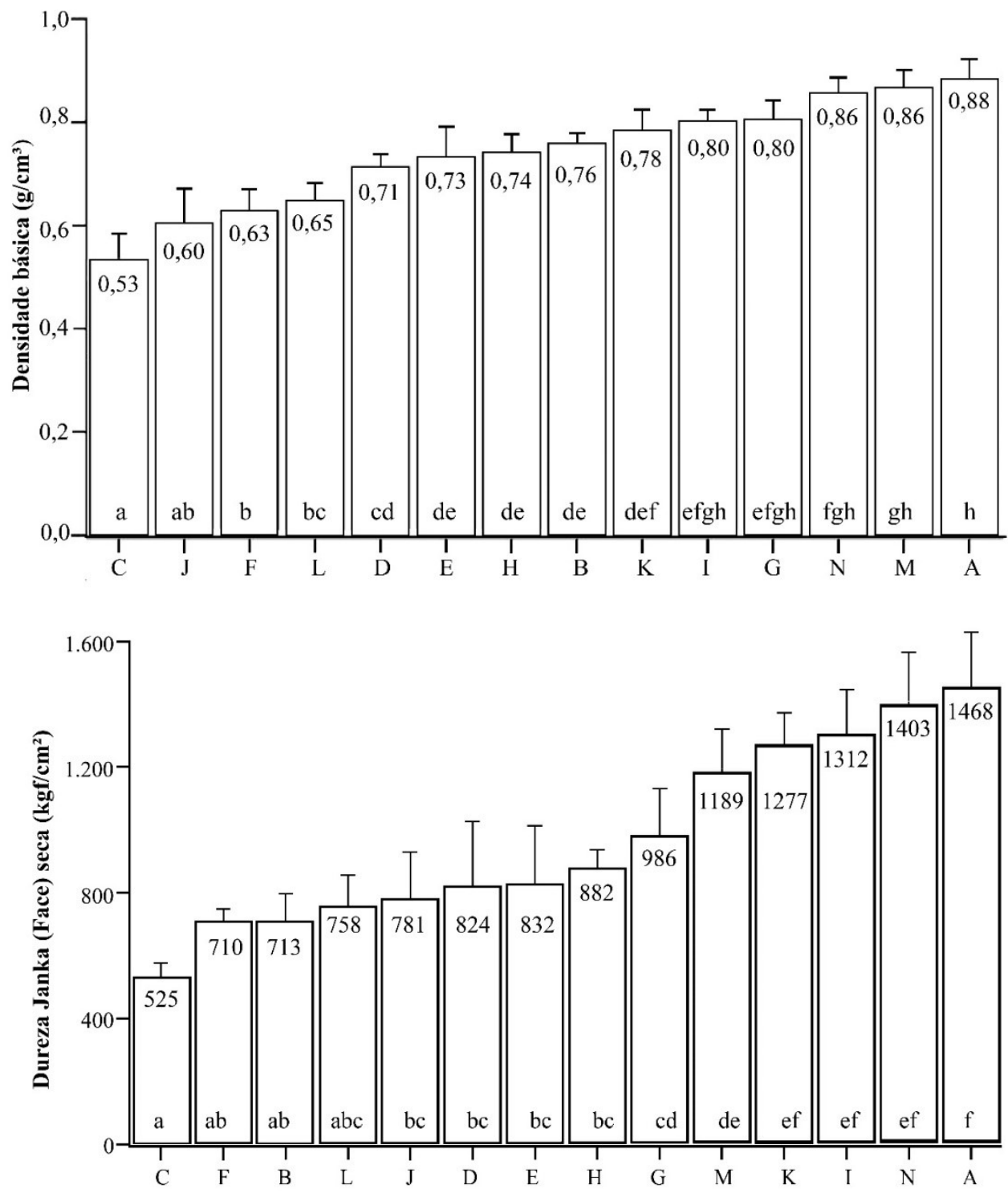


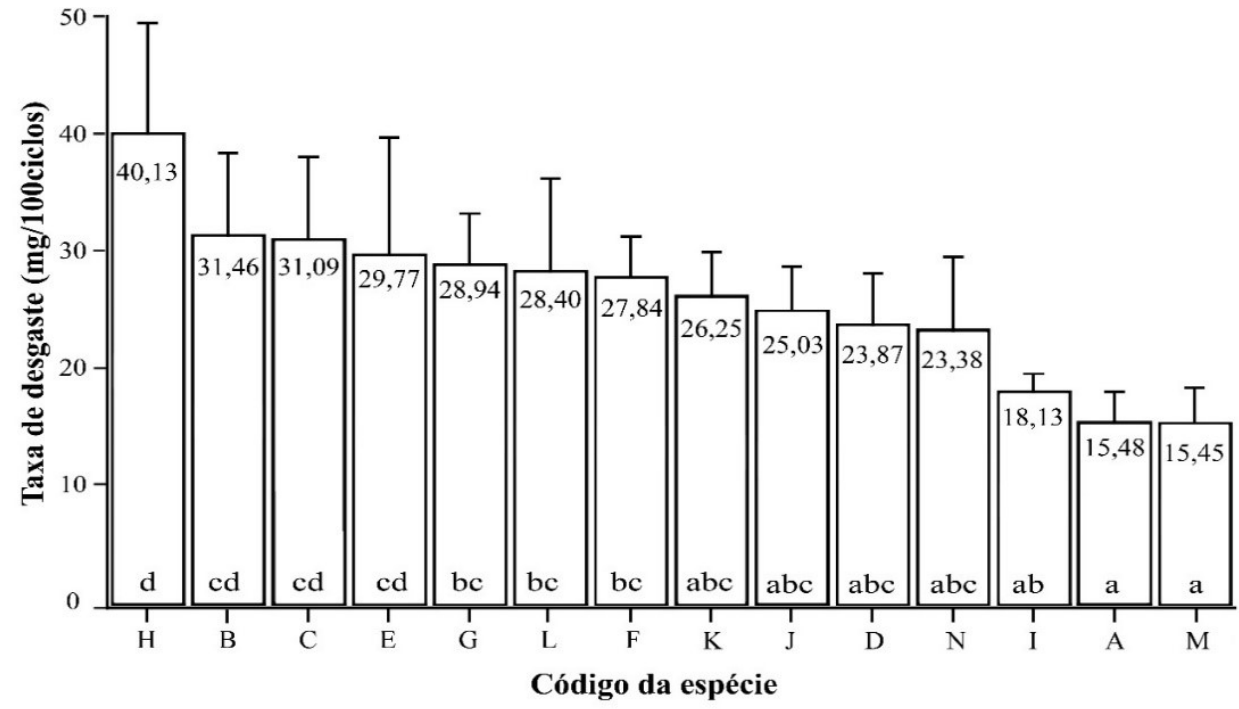

Figura 2. Densidade básica, dureza Janka na face seca e taxa de desgaste à abrasão das espécies de madeiras. (Barras em "I" são desvio padrão; Dureza Janka a 12\% de umidade; letras minúsculas iguais em cada gráfico não diferem a 5\% de significância pelo teste de Tukey).

Figure 2. Basic density, side Janka hardness and wear rate of the wood species evaluated. (Bars in "I" are standard deviation; Janka Hardness at 12\% MC; same lower case letter in each graph do not differ at 5\% significance level of Tukey test).

apresentados por Lopes (2012), e são compatíveis com os resultados do presente trabalho. De acordo com o autor, madeiras de Gombeira (Swartzia laurifolia Benth.) e Favaparicá (Balizia elegans (Ducke) Barneby \& J.W.Grimes) apresentaram menor e maior desgaste, respectivamente. Jankowska et al. (2015) avaliou o efeito do intemperismo acelerado na resistência a abrasão de Garapa (Apuleia leiocarpa), Cumaru (Dipteryx odorata) e Tatajuba (Bagassa guianensis). Com densidade aparente (a 12\% de teor de umidade) entre 0,82 e $0,905 \mathrm{~g} / \mathrm{cm}^{3}$, essas espécies apresentaram dureza Janka entre 499 e $773 \mathrm{kgf} / \mathrm{cm}^{2}$ e, desgaste à abrasão, entre 14 e 39 mg/100ciclos, bem similares aos deste estudo.

Os valores de dureza Janka entre 525 e $1468 \mathrm{kgf} / \mathrm{cm}^{2}$, aliado ao fato de serem espécies de média para alta densidade básica, de acordo com a classificação sugerida por Melo et al. (1990), qualificam todas as espécies como potencial para uso em pisos. O valor máximo de dureza encontrado por
Swaczyna et al. (2011), foi de $431 \mathrm{kgf} / \mathrm{cm}^{2}$ para madeiras de Oak, Mahogany e Pine usadas em pisos na Polônia.

\section{Correlação da abrasão com outras propriedades}

A Tabela 2 mostra que a correlação de Pearson entre a resistência à abrasão (TD) e as demais propriedades citadas é negativa e varia entre $42,4 \%$ e $58,9 \%$. Esse resultado sugere que não é possível obter uma equação com alta precisão para estimar o desgaste abrasivo em função apenas das propriedades citadas.

A resistência à abrasão, representada pela taxa de desgaste, apresentou maiores correlações com a dureza Janka de topo na condição seca $(-0,589)$, de face seca $(-0,538)$ e na extração de prego de face seca $(-0,578)$ e, menor relação com a densidade básica $(-0,401)$, conforme a Tabela 2 . Swaczyna et al. (2011), obtiveram correlações entre densidade seca e dureza de 0,69 e resistência à abrasão e dureza de $-0,41$, todas

Tabela 2. Análise de correlação de Pearson entre resistência à abrasão, densidade básica, densidade aparente, dureza Janka e extração de prego. 
Table 2. Correlation of Pearson between abrasion resistance, basic density, apparent density at 12\%, Janka hardness and nail extraction.

\begin{tabular}{|c|c|c|c|c|c|c|}
\hline Propriedade & $\begin{array}{l}\text { Densidade } \\
\text { Aparente }^{1}\end{array}$ & $\begin{array}{c}\text { Densidade } \\
\text { Básica }\end{array}$ & $\begin{array}{c}\text { Dureza Face } \\
\text { Seca }^{1}\end{array}$ & $\begin{array}{c}\text { Dureza } \\
\text { Topo Seca }{ }^{1}\end{array}$ & $\begin{array}{c}\text { Prego } \\
\text { Face Seca }\end{array}$ & $\begin{array}{c}\mathrm{TD}^{2} \\
\text { (Abrasão) }\end{array}$ \\
\hline Densidade Aparente $^{1}$ & 1 & & & & & \\
\hline Densidade Básica & $0,964^{\star \star}$ & 1 & & & & \\
\hline Dureza Face Seca & $0,850^{* *}$ & $0,810^{\star *}$ & 1 & & & \\
\hline Dureza Topo Seca & $0,774^{* *}$ & $0,722^{\star *}$ & $0,970^{* *}$ & 1 & & \\
\hline Prego Face Seca & $0,334^{\star *}$ & $0,275^{\star}$ & $0,639^{\star *}$ & $0,750^{\star *}$ & 1 & \\
\hline $\mathrm{TD}^{2}$ (Abrasão) & $-0,424^{\star *}$ & $-0,401^{\star *}$ & $-0,538^{\star *}$ & $-0,589^{* *}$ & $-0,578^{\star *}$ & 1 \\
\hline
\end{tabular}

$\left({ }^{1}\right)$ a $12 \%$ de teor de umidade; $\left({ }^{2}\right) \mathrm{TD}=$ taxa de desgaste; $\left({ }^{*}\right)$ correlação significativa a 5\%; $\left.{ }^{* *}\right)$ correlação significativa a $1 \% ; n=84$.

inferiores aos valores do presente estudo.

A correlação com dureza Janka encontrada nesse trabalho foi significante e negativa e indica que é viável a condução de maiores estudos nesse sentido, focados em outras propriedades importantes da madeira, ou seja, na anatomia e nas propriedades químicas.

\section{Conclusões}

Os resultados de resistência à abrasão mostram que as madeiras estudadas são altamente resistentes e apropriadas para pisos.

Dentre as espécies, destacam-se com as melhores propriedades: Angelim vermelho (A), Sucupira preta (I), Sapucaia (M) e Jarana amarela (N).

Apesar das correlações obtidas nesse trabalho, recomenda-se a realização de estudos complementares que considere outras propriedades da madeira, como arranjo o anatômico e a contração e o inchamento, essenciais na confecção de assoalhos, com objetivo de determinar se essas correlações podem ser aumentadas.

\section{Agradecimentos}

Os autores agradecem ao Serviço Florestal Brasileiro e ao Conselho Nacional de Pesquisa e Desenvolvimento (CNPq) pelo apoio financeiro em forma de bolsa de iniciação científica.

\section{Referências}

AMERICAN SOCIETY FOR TESTING AND MATERIALS. ASTM D143-14: Standard Test Methods for Small Clear Specimens of Timber. Book of Standards, v. 04.10. Philadelphia, 31p., 2014.

AMERICAN SOCIETY FOR TESTING AND MATERIALS. ASTM D4060-14: Standard Test Method for Abrasion Resistance of Organic Coatings by the Taber Abraser. Book of Standards, v. 04.10. Philadelphia, 5p., 2014.

ANDRADE, A. Guia básico para instalação de pisos de madeira. 2 ed. - Piracicaba: ANPM, 104p., 2015.

ASSOCIAÇÃO BRASILEIRA DE NORMAS TÉCNICAS ABNT. NBR 14535-2008: Móveis de madeira - Tratamento de superfícies - Requisitos de proteção e acabamento. Rio de Janeiro, 22 p., 2008.

CADEMARTORI, P.H.G. de; NISGOSKI, S.; MAGALHÃES, W.L.E.; MUNIZ, G.I.B. de. Surface wettability of Brazilian tropical wood flooring treated with He plasma. Maderas. Ciencia y tecnología, v. 18, n. 4, p. 715-722, 2016. 
JANKOWSKA, AGNIESZKA; ZURAWSKI, PAWEL; MAZUREK, ANDRZEJ. The influence of artificial weathering on abrasion resistance of selected wood species from South America. Forestry and Wood Technology, v. 89, p. $60-65,2015$.

LOPES, M.D.M. Resistência à abrasão de onze espécies de madeira da floresta Amazônica. In: XIII ENCONTRO BRASILEIRO EM MADEIRAS E EM ESTRUTURAS DE MADEIRA, 13, UFES/Vitória, 2012.

MELO, J.E.; CORADIN, V.T.R.; MENDES, J.C. Classes de densidade de madeira para a Amazônia brasileira. In: ANAIS DO CONGRESSO FLORESTAL BRASILEIRO, 6: 695-699. São Paulo, SP, Brasil. 1990.

NCUBE, E. Use of a simple abrasive-wear resistance test device to assess the suitability of selected hardwoods for wood flooring. Scientific Research and Essay, v.3, n. 5, p. 168-173, 2008.

PAES, J.B.; OLIVEIRA, A.K.F. de; LEAL, A.F. de; NASCIMENTO, J.W.B do. Caracterização e aspecto de um piso confeccionado com bambu (Dendrocalamus giganteus Munro). Ciência da Madeira, v. 01, n. 1, p. 52-67, 2010.

SILVA, D.F.; BITTENCOURT, M.R. Estudo do desgaste à abrasão do eucalipto, madeira laminada e bambu gigante laminado utilizados como elemento de piso. In: VIII ENCONTRO BRASILEIRO EM MADEIRAS E EM ESTRUTURAS DE MADEIRA, 7, Uberlândia, 2002.

SWACZYNA, I.; KĘDZIERSKI, A.; TOMUSIAK, A.; CICHY, A.; RÓŻAŃSKA, A. Hardness and wear resistance tests of the wood species most frequently used in flooring panels. Forestry and Wood Technology, v. 76, p. 82-87, 2011. 